Review Article

\title{
Immunotherapeutic Approaches in Triple-Negative Breast Cancer: State of the Art and Future Perspectives
}

\author{
Karima Oualla $\mathbb{D}^{1},{ }^{1}$ Loay Kassem $\mathbb{D},{ }^{2}$ Lamiae Nouiakh, ${ }^{1}$ Lamiae Amaadour $\mathbb{D},{ }^{1}$ \\ Zineb Benbrahim $\left({ }^{1},{ }^{1}\right.$ Samia Arifi, ${ }^{1}$ and Nawfel Mellas $\left({ }^{1}{ }^{1}\right.$ \\ ${ }^{1}$ Medical Oncology Department, Hassan II University Hospital, Sidi Mohamed Ben Abdellah University, Fes, Morocco \\ ${ }^{2}$ Clinical Oncology Department, Kasr Al-Ainy School of Medicine, Cairo University, Giza, Egypt \\ Correspondence should be addressed to Karima Oualla; karimarauf@hotmail.com
}

Received 15 May 2020; Accepted 16 June 2020; Published 5 November 2020

Academic Editor: Debra A. Tonetti

Copyright (C) 2020 Karima Oualla et al. This is an open access article distributed under the Creative Commons Attribution License, which permits unrestricted use, distribution, and reproduction in any medium, provided the original work is properly cited.

\begin{abstract}
Triple-negative breast cancer (TNBC) is characterized by the absence of estrogen receptor (ER), progesterone receptor (PR), and human epidermal growth factor receptor 2 (HER2). It accounts for $15 \%-20 \%$ of all breast cancers and is associated with an aggressive evolution and poor outcomes with the majority of recurrences and deaths occurring in the first 5 years. Chemotherapy remains the mainstay of treatment in the absence of effective targets, but the good understanding of immune tumor microenvironment, the identification of immune-related targets, and the role of tumor-infiltrating lymphocytes (TILs) in TNBC has allowed to develop promising immunotherapeutic strategies for this unique subset of breast cancer. Recently, immunotherapy is being extensively explored in TNBC and clinical trials have shown promising results. In this article, we tried to explain the rationale and mechanisms of targeting the immune system in TNBC, to report the results from recent clinical trials that put immunotherapy as a new standard of care in TNBC in addition to ongoing trials and future directions in the next decade.
\end{abstract}

\section{Overview}

Triple-negative breast cancer (TNBC) is a molecular subtype of breast cancer characterized by the absence of expression of estrogen receptor (ER), progesterone receptor (PR), and human epidermal growth factor receptor 2 (HER2) [1]. TNBC tends to occur more in young women $(<40$ years old) and typically presents with aggressive biology including high-grade invasive ductal carcinomas and high proliferative rate [2]. It also presents a particular pattern of metastases with higher rates of visceral and brain metastases, and poor outcomes with early recurrences and shorter survival [2].

At the molecular level, distinct intrinsic subtypes of TNBC were distinguished using gene expression signatures [3]. Gene expression profiling of TNBCs has identified six molecular subtypes including two basal-like subtypes (BL1 and BL2), mesenchymal (M), mesenchymal stem-like (MSL), immunomodulatory (IM), and a luminal androgen receptor (LAR) $[4,5]$. This genomic profiling allowed con- ducting several researches aiming at developing more personalized treatments for TNBC patients.

Therapeutically, for many decades, cytotoxic chemotherapy was the mainstay treatment in the absence of actionable targets with short survival [6]. Then recently, it has been shown that the immune system has an important role in tumor initiation and progression of breast cancer and also in the destruction of cancer cells [7]. The genetic and epigenetic alterations in TNBC lead to tumor-associated antigens that allow the immune system to recognize tumor cells from normal cells. The immune system blocks the development and progression of cancer cells via tumor-directed immune responses involving mainly $\mathrm{T}$ lymphocytes.

Active immunotherapy using immune checkpoint inhibitors (ICIs) acts by enhancing the activity of the immune system via disrupting negative immune regulators to enhance the immune response [8]. Therefore, different ICIs have been first explored in cancers considered as highly immunogenic such as melanoma, lung cancer, and renal cancer. ICIs have 
shown impressive results which made them new standards of care in the treatment of early and advanced stages of different cancers including bronchial cancers, melanomas, and urothelial cancers. These impressive results led to investigating the role of ICIs in breast cancer particularly in the TNBC subtype.

\section{Immunogenicity of TNBC}

Unlike melanoma, lung cancer, and kidney cancer, breast cancer was considered as a nonimmunogenic cancer for long decades with low $\mathrm{T}$ cell infiltration. However, in contrast to the other breast cancer subtypes, TNBC is characterized by higher tumor immune infiltrate and higher degree of stromal and tumor-infiltrating lymphocytes (TILs) [9]. Several studies have shown that the TILs were associated with better prognosis and higher response to therapy in breast cancer especially in TNBC $[10,11]$.

Additionally, further studies have suggested activation of inhibitory immune checkpoints (as CTLA-4 and PD-1/PDL1 axis) in TNBCs with higher PD-L1 expression in comparison with luminal subtypes. The inhibitory action of PD-1 bound to its ligand (PD-L1) suppresses the immune response in cancer cells. Therefore, the upregulation of PD-L1 expression in tumor cells allows an evasion of the tumor cells from the immune system detection, which subsequently leads to tumor progression [12].

PD-L1 expression inhibits different immune cells in the tumor microenvironment including $\mathrm{T}$ cells, B cells, natural killer cells, dendritic cells, and macrophages, suggesting that PD-1 expression is a mechanism of restriction of immunity, provided through the innate and adaptive immune system. PD-1 has a major role in the negative regulation of $\mathrm{T}$ cell activity by blocking $\mathrm{T}$ cells and modulating immune response [13].

Moreover, TNBC is characterized by a high mutational burden that provides genomic instability and subsequently leads to higher production of neoantigens which make TNBC more immunogenic than other breast cancer subtypes.

Several factors intervene in the antitumor immune response. The cytotoxic CD8-positive T lymphocytes, type 1 macrophages, and intratumoral $B$ cells play a crucial role in the antitumor microenvironment. Additionally, FOXP3 ${ }^{+-}$ $\mathrm{CD}^{+}$regulatory T cells (Tregs) are mediators of immune tolerance, and therefore, they are associated with poor outcomes [13]. The PD-L1 was reported to be expressed in around $20 \%$ to $30 \%$ of TNBC and was correlated with aggressive characteristics including higher grade and high proliferation rate. $\mathrm{PD}-1$ interacts with its ligand $\mathrm{PD}-\mathrm{L} 1$, and this interaction on $\mathrm{T}$ cells is a major mechanism of tumor immune evasion and leads to the suppression of antitumor immunity by exerting a negative regulation on T cells, cytolytic activity, and production of cytokine. Subsequently, the blockade of these targets leads to increasing the antitumor immune response by the blockade of immune-regulating proteins that downregulate the immune system [14].

CTLA4 also has an important role in regulating immune responses early in the process of $\mathrm{T}$ cell activation. Therefore, its inhibition by monoclonal antibodies blocks the interac- tion between T cells and the receptor via CD28 on its cell surface. This blockade increases the ratio of $\mathrm{CD}^{+} \mathrm{T}$ cells to Foxp $3^{+} \mathrm{T}$ regulatory cells, promotes the antitumor activity of $\mathrm{CD}^{+} \mathrm{T}$ cells, and blocks the suppressive function of T regulatory cells $[13,14]$.

These results, put together, show that immunotherapy is a promising modality in TNBC, and the use of anti-PD1/PD-L1 in the treatment of TNBC must receive much attention.

\section{Clinical Trials with Immune Checkpoint Inhibitors in TNBC (Table 1)}

\subsection{PD-1 Inhibitors}

3.1.1. Pembrolizumab. Pembrolizumab, a PD-1 inhibitor, has been evaluated as monotherapy in PD-L1-positive heavily pretreated metastatic TNBC patients [15]. In this phase $1 \mathrm{~b}$ trial, $47 \%$ of patients had received more than 3 lines of treatment and $21.9 \%$ had received 5 or more treatments. The results revealed an objective response rate (ORR) of $18.5 \%$, a partial response in $14.8 \%$ of patients, a complete response (CR) in $3.7 \%$ of cases, and $25.9 \%$ had a stable disease (SD). The overall survival (OS) rate at 2 years was $22 \%$ [15]. It can be explained by the maintained disease control seen in good responders to therapy.

Another phase single-arm multicohort II study (KEYNOTE-086) has investigated the role of pembrolizumab in monotherapy in pretreated metastatic TNBC [16]. Cohort A included 170 TNBC patients who received one or more systemic therapy. The endpoint was to assess the efficacy and the safety of pembrolizumab independently of PD-L1 expression. The primary endpoints were the ORR in the total and PD-L1-positive populations and safety. Sixty percent of patients had PD-L1-positive tumors. After a median followup of 11.9 months, the ORR was $5 \%$ and the disease control rate was $8 \%$. The response rate was not impacted by PD-L1. The median PFS was 2.0 months with an estimated 6month PFS rate of $12 \%$ [16]. Cohort B of the same study included 52 patients and investigated the safety and efficacy of pembrolizumab in patients with TNBC with no prior systemic treatment for tumors with PD-L1-positive tumors defined by an IHC-based composite score. The ORR was $23 \%$ after a median follow-up of 7 months, and the median PFS was 2.1 months (95\% CI 2.0-3.9); the estimated 6month PFS rate was 29\%, and the median duration of response was 8.4 months [17]. The improved response in cohort B may be a result of the use of pembrolizumab as a first-line treatment and the selection of only PD- $\mathrm{L}^{+}$tumors as an inclusion criterion.

A phase Ib/II trial has assessed the combination of pembrolizumab with eribulin mesylate in patients with metastatic disease, treated with at least 2 prior lines of chemotherapy [18].

The findings revealed an ORR of $25.6 \%$ in the 82 evaluated patients and $30.5 \%$ of the clinical benefit rate. Response was regardless of PD-L1 expression $(25.7 \%$ and $25.0 \%$ in the PD-L1-positive and PD-L1-negative cohorts, respectively) [18]. The combination also resulted in improved PFS and 


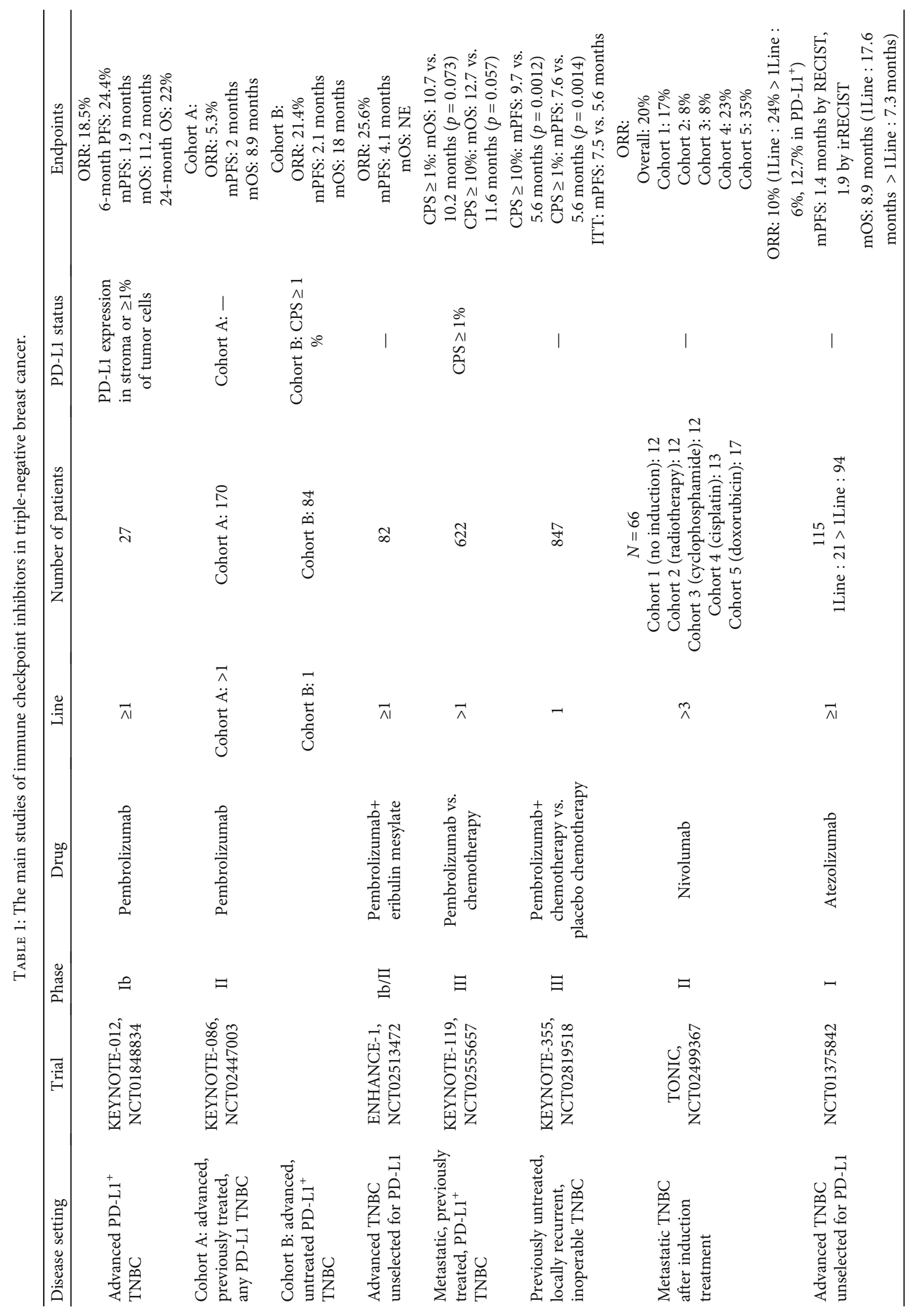




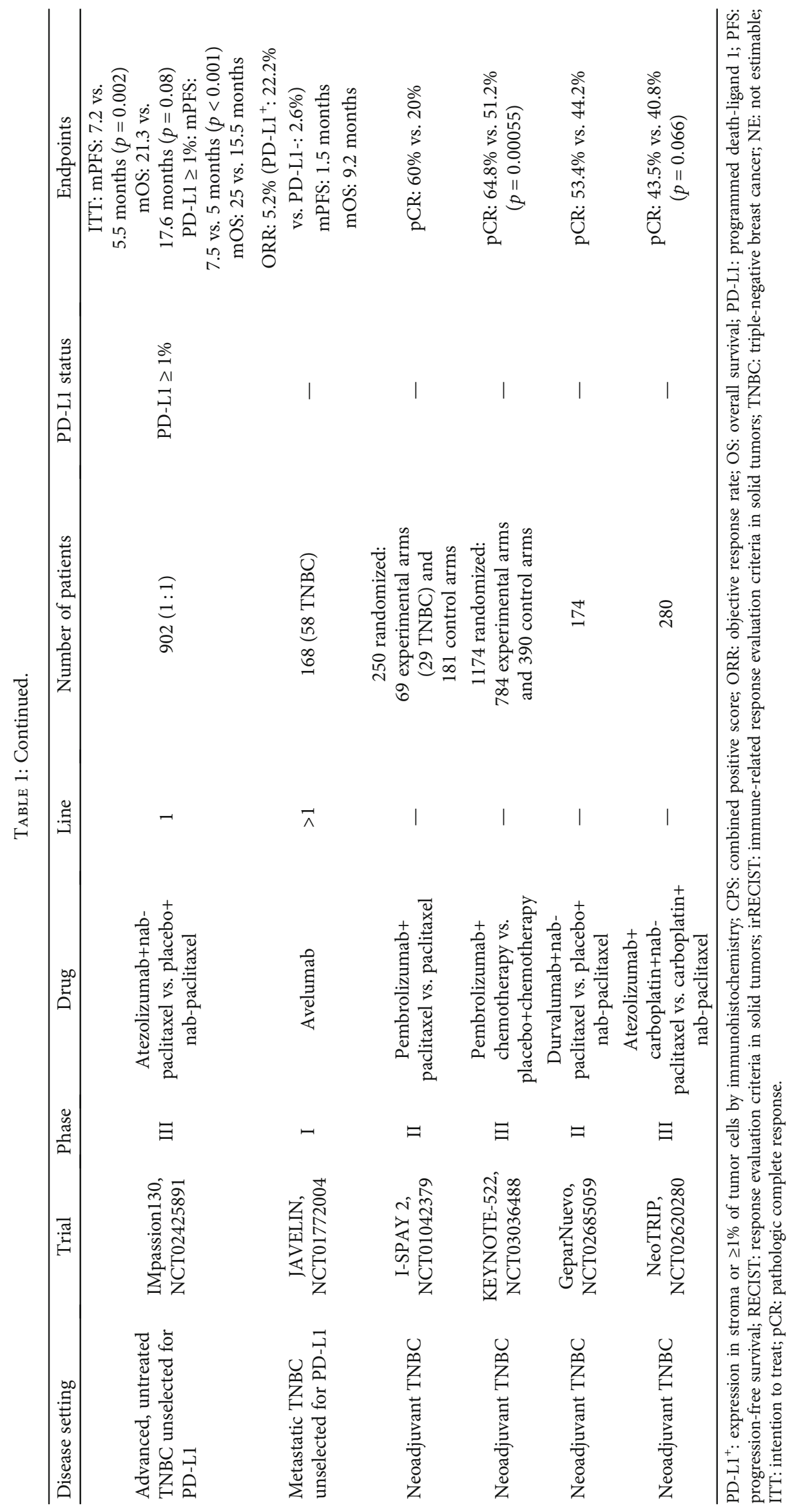


OS independently of PD-L1 status, and the safety profile was comparable to monotherapy. Further exploration of this combination is needed.

KEYNOTE-119 is a phase III study that has evaluated pembrolizumab versus single-agent chemotherapy per investigator's choice (capecitabine, eribulin, gemcitabine, or vinorelbine) in 662 patients with metastatic TNBC who progressed on 1 or more lines of chemotherapy. The study did not meet its prespecified primary endpoint of superior OS compared to standard chemotherapy. The trial did not show any new safety concerns [19].

Another global phase III trial (KEYNOTE-355, NCT02819518) has assessed the combination of pembrolizumab with chemotherapy versus placebo with chemotherapy in patients with previously untreated, locally recurrent, inoperable TNBC. Pembrolizumab plus chemotherapy significantly improved PFS vs. chemotherapy alone when CPS $\geq 10$ which is one of the primary objectives. Despite the boundary for a statistically significant benefit of the combination in patients with CPS $\geq 1$ tumors was not met and formal testing in ITT was not performed, the pembrolizumab treatment efficacy was higher with PD-L1 enrichment. OS follow-up is ongoing, and no new safety concerns were reported [20].

The promising results in metastatic stages of TNBC provided enough evidence to conduct studies with pembrolizumab in early stages. Among them, the I-SPY 2 phase II multicenter trial evaluated the addition of pembrolizumab to standard neoadjuvant chemotherapy based on paclitaxel followed by doxorubicin and cyclophosphamide. This study showed that the combination [19] was likely to result in a significant improvement of pCR in TNBC (60\% vs. $20 \%)$ [21].

More recently, the KEYNOTE-522 phase III study has assessed the addition of pembrolizumab [19] to neoadjuvant chemotherapy for stages IIa to IIIb TNBC [22]. Patients were randomized 2:1 to pembrolizumab or placebo. Both arms were given with 4 cycles of paclitaxel+carboplatin and then with 4 cycles of doxorubicin or epirubicin+cyclophosphamide. In adjuvant setting after curative surgery, patients received pembrolizumab or placebo for 9 cycles or until relapse or unacceptable toxicity. Primary endpoints were the pathologic complete remission rate (pCR) and eventfree survival (EFS).

After a median follow-up of 15.5 months, adding pembrolizumab significantly improved $\mathrm{pCR}$ compared to chemotherapy alone: $64.8 \%$ vs. $51.2 \%(p=0.00055)$. For pembrolizumab vs. placebo, pCR was $68.9 \%$ vs. $54.9 \%$ in the PD- $\mathrm{L1}^{+}$population and $45.3 \%$ vs. $30.3 \%$ in the PD-L1 population. Additionally, the pembrolizumab arm also showed a favorable trend in EFS (HR $=0.63$ [95\% CI 0.43-0.93]). Regarding safety, grade 3 or higher treatment-related adverse event rates were $78.0 \%$ in the pembrolizumab plus chemotherapy group and $73 \%$ in the placebo+chemotherapy group (death incidence, $0.4 \%$ vs. $0.3 \%$, respectively) [22].

Another ongoing phase III trial (SWOG-S1418, BR006; NCT02954874) is evaluating the efficacy and safety of pembrolizumab as adjuvant therapy for TNBC with $\geq 1 \mathrm{~cm}$ residual invasive cancer or positive lymph nodes $\left(\mathrm{ypN}^{+}\right)$after neoadjuvant chemotherapy.
3.1.2. Nivolumab. Nivolumab was assessed in several phase I and II trials of TNBC patients. The TONIC trial is an adaptive phase II randomized noncomparative trial that evaluated nivolumab in patients with metastatic TNBC after induction treatment including radiation, low-dose doxorubicin, metronomic cyclophosphamide, and cisplatin [23]. The objective response rate (ORR) per RECIST v1.1 with nivolumab for the whole cohort was $22 \%$ and $24 \%$ for iRECIST, which included 1 (2\%) CR, and 11 (22\%) PR. Additionally, stable disease (SD) lasting more than 24 weeks was achieved in 1 (2\%) patient, which resulted in a $26 \%$ clinical benefit rate $(\mathrm{CBR}=\mathrm{CR}+\mathrm{PR}+\mathrm{SD}>24$ weeks). The median duration of response was 9 months (95\% confidence interval (CI) 5.5NA). Preliminary analyses showed that the response rate might be higher after induction therapy with doxorubicin or cisplatin and those patients with higher leukocyte infiltration and CD8 $\mathrm{T}$ cell counts were better responders to treatment [23].

Another ongoing phase II study is evaluating carboplatin with or without nivolumab in metastatic TNBC (NCT03414684).

\subsection{PD-L1 Inhibitors}

3.2.1. Atezolizumab. Atezolizumab is a monoclonal antibody that binds selectively to PD-L1 on immune cells or tumor cells to prevent interactions with the $\mathrm{PD}-1$ receptor or B71. A phase I study of single-agent atezolizumab included 116 patients with metastatic TNBC irrespective of prior therapy or PD-L1 status [24]. The primary endpoints were safety and tolerability. The ORR was $10 \%$ in the overall population and $24 \%$ in those receiving atezolizumab as first-line treatment. Interestingly, the median duration of response was 21 months (range, 3 to $\geq 38$ ). Of note, liver metastases, LDH levels, tumor burden, and performance status were associated with worse outcomes. Treatment-related adverse events were mostly grades $1-2$ (in $79 \%$ of cases) with the most common being fever, fatigue and nausea, diarrhea, and pruritus [24].

Important results were presented from the phase III IMpassion 130 trial. This study included 902 patients with treatment-naïve metastatic TNBC who were randomly assigned to receive nab-paclitaxel alone or in combination with atezolizumab. The combination reduced the risk of disease progression or death by $20 \%$ in all patients and by $38 \%$ in the subgroup expressing PD-L1 which accounted for $41 \%$ of all patients [25].

After a median follow-up of slightly more than 1 year, the median progression-free survival (PFS) was 7.2 months with the combination compared with 5.5 months for placebo plus nab-paclitaxel in the intention-to-treat analysis (hazard ratio (HR) for progression or death $=0.80 ; p=0.002$ ). Patients with PD-L1-positive tumors who received the combination had a $38 \%$ reduction in the risk of progression and death compared with nab-paclitaxel alone $(\mathrm{HR}=0.62 ; 7.5$ vs. 5.0 months; $p<0.001$ ).

At first interim analysis, in the intent-to-treat group, median OS was 21.3 months with the combination compared with 17.6 months for placebo plus nab-paclitaxel $(\mathrm{HR}=0.84$; 
$p=0.08)$. Among those with PD-L1-positive tumors, the median overall survival was 25.0 months compared with 15.5 months, respectively. The safety of atezolizumab plus nab-paclitaxel was consistent with the known toxic effects of each agent [25].

After these results, the Food and Drug Administration (FDA) granted accelerated approval to atezolizumab in March 2019, and atezolizumab in combination with nabpaclitaxel became the new upfront standard of care in the subset of patients with unresectable locally advanced or metastatic PD-L1-positive TNBC.

Another phase III trial is evaluating atezolizumab in combination with paclitaxel compared with placebo with paclitaxel for patients with previously untreated inoperable locally advanced or metastatic TNBC (NCT03125902) [26]. In the neoadjuvant setting, a phase III randomized study is investigating the efficacy and safety of atezolizumab in combination with neoadjuvant anthracycline/nab-paclitaxelbased chemotherapy compared with placebo and chemotherapy (NCT03197935) [27]. Another phase III neoadjuvant trial is studying the efficacy of atezolizumab in locally advanced TNBC patients undergoing treatment with nabpaclitaxel and carboplatin. The addition of atezolizumab to neoadjuvant chemotherapy did not improve pathologic complete response rates in early, high-risk triple-negative breast cancer. In this trial, PD-L1 expression correlated with pathologic complete response [28].

3.2.2. Avelumab. Avelumab is another $\mathrm{PD}-\mathrm{L} 1$ inhibitor undergoing clinical development. In a phase Ib solid tumor basket trial (JAVELIN; NCT01772004), avelumab was used to treat an expansion cohort consisting of 168 metastatic breast cancer patients, with tumors that were unselected for PD-L1 status and breast cancer subtype [29]. The TNBC subtype represented $34.5 \%$ of patients. Approximately $50 \%$ of the TNBC patients had $\leq 1$ prior regimen for metastatic disease. The ORR in the TNBC cohort was $8.6 \%$ (95\% CI 2.919); 5 of 58 patients had a PR, and 13 had stable disease (22.4\%) [29].

Avelumab is also under assessment in adjuvant setting in the A-BRAVE Trial (NCT02926196). It is a phase III randomized trial evaluating adjuvant treatment with avelumab in 335 patients with TNBC who completed definitive curative therapy [30].

3.2.3. Durvalumab. In metastatic TNBC, durvalumab is being evaluated in combination with Vigil (autologous tumor cell immunotherapy; NCT02725489) [31] and in combination with paclitaxel (NCT02628132) [32], olaparib (NCT02484404) [33], tremelimumab (NCT02527434) [34], and epacadostat, an inhibitor of indoleamine 2,3-dioxygenase (NCT02318277) [35].

It was also tested in combination with other agents for early-stage TNBC.

The addition of durvalumab to anthracycline/taxanebased chemotherapy showed encouraging results as neoadjuvant therapy for early TNBC in the randomized phase II GeparNuevo study [36]. The primary endpoint was the pCR rate that was $53.4 \%$ in the durvalumab arm vs. $44.2 \%$ for chemotherapy alone (control arm), and the best response rates were observed when durvalumab was given for a window of 2 weeks before chemotherapy, priming the immune system first.

Another phase I/II neoadjuvant study (NCT02489448) of weekly nab-paclitaxel followed by dose-dense doxorubicin and cyclophosphamide with concurrent durvalumab in stages I-III TNBC showed that addition of durvalumab is safe and the pCR rates appear to be higher than what is expected with chemotherapy alone [37].

\section{Future Perspectives}

4.1. Combining Immune Checkpoint Inhibitors with PARP Inhibitors. So far, few clinical trials have explored the immunomodulatory potential of PARPi. However, recent data demonstrate the presence of a functional link between DNA damage response and anticancer immunity [38]. This link could be explained by the impact of genomic instability and tumor mutational burden on the production of tumor neoantigens and also by the immunogenic cell death induced by DNA damage.

Additionally, the activation of the "innate cytosolic immunity" signaling pathways is in response to DNA damage. This latter aspect was notably revealed by two recent studies which have demonstrated the capacity of certain DNA repair defects, present in the tumor, to stimulate an antitumor immune response by activation of the cGAS/STING (cyclic) pathway and GMP-AMP synthase/stimulator of interferon genes [39, 40].

Several trials have been conducted to explore the real effectiveness of combination therapy.

TOPACIO/KEYNOTE-162 is an open-label, single-arm phase 2 trial including 55 patients with advanced TNBC regardless of the BRCA mutation status or the programmed expression of the death ligand 1 (PD-L1) having received niraparib and pembrolizumab; the ORR was $21 \%$ and the disease control rate was $49 \%$. In 15 patients with a BRCA tumor mutation, the ORR was $47 \%$ and the disease control rate was $80 \%$ [41].

Many other trials are assessing the combination of immune checkpoint inhibitors and PARP inhibitors.

A randomized phase II study is exploring the efficacy of olaparib or olaparib in combination with durvalumab in platinum-treated mTNBC NCT03167619 [42].

Another phase I/II is evaluating durvalumab in combination with olaparib and/or cediranib NCT02484404 [33].

Avelumab, another anti PD-L1, is under evaluation in association with talazoparib in phase II trial NCT03330405 [43].

Additionally, atezolizumab is also being explored with rucaparib in a phase I study NCT03101280 [44] and with olaparib in a phase II trial NCT02849496 [45].

4.2. Combining Immune Checkpoint Inhibitors with CDK4/6 Inhibitors. CDK4/6 inhibitors have been shown to be able to induce an antitumor immune response by different mechanisms, including increased presentation of the antigen by tumor cells, in addition to stimulation of the activation of 
effector $\mathrm{T}$ lymphocytes and reduction of proliferation of immunosuppressive Treg cells $[46,47]$.

The combination of CDK4/6 inhibitors with immune checkpoint inhibitors, targeting PD-1 and CTLA-4, has shown promising results in preclinical trials by inducing complete and lasting regressions ( $>1$ year) in mouse models of established xenografts of human TNBC [48].

The rationale of the efficacy of the combination of CDK4/6 inhibitors and immunotherapy was based on different mouse models. Preliminary positive results from a phase Ib clinical trial studying abemaciclib with pembrolizumab in HER2-negative ER-positive MBCs showed an ORR of $14.3 \%$ in a 16 -week interim analysis with a $75 \%$ of disease control rate [49]. Randomized clinical studies investigating the combination of CDK4/6 inhibitors and immunotherapy in the triple-negative subgroup are needed to shed light on the effectiveness of this strategy in this group of patients.

4.3. Adoptive T Cell Therapy. CAR $\mathrm{T}$ cells are genetically engineered $\mathrm{T}$ cells equipped with a tumor-specific chimeric antigen receptor. Antibody-derived chimeric antigen receptor (CAR) T cell therapy has demonstrated very promising results in hematologic malignancies with less success in solid tumors. Some research has shown that CAR T cells targeting tumor MUC1 glycoprotein may reduce the growth of TNBC [50]. The monoclonal antibody, TAB004, specifically recognizes the aberrantly glycosylated tumor form of MUC1 in all subtypes of breast cancer including 95\% of TNBC while sparing recognition of normal tissue MUC1. MUC28z CAR $\mathrm{T}$ cells demonstrated significant target-specific cytotoxicity against a panel of human TNBC cells. Thus, MUC28z CAR $\mathrm{T}$ cells have high therapeutic potential against tMUC1positive TNBC tumors with minimal damage to normal breast epithelial cells [50].

Additionally, encouraging results were seen with TGF- $\beta$ that suppresses cytolytic capacity, cytokine production, and proliferation of CAR T cells against TNBC in vitro. These immunosuppressive effects can be neutralized by TGF- $\beta$ receptor I kinase inhibition. Therefore, these findings encourage the evaluation of ROR $1^{+}$CAR $\mathrm{T}$ cells against TNBC in combination with a TGF- $\beta$ inhibitor [51].

More recently, mesothelin, a cell surface glycoprotein normally present in mesothelial cells, was identified as a potential immunotherapy target [52]. This glycoprotein has already demonstrated encouraging results in mesothelioma and ovarian cancer. In a study including 99 primary breast cancers, it has been found that $67 \%$ of patients with TNBC expressed the mesothelin in at least $5 \%$ of tumor cells, with $19 \%$ of patients with TNBC expressing mesothelin in over $50 \%$ of tumor cells. In contrast, it was rarely expressed in luminal or HER2-positive breast cancer [52, 53]. These findings showed that mesothelin can be a promising target for adoptive $\mathrm{T}$ cell therapy of TNBC, but more advanced research is still needed to validate it.

Another molecule is under investigation in TNBC, which is an intercellular adhesion molecule-1 (ICAM-1). It has been found that this molecule is upregulated in TNBC and could and then may be a promising target in the future by evaluat- ing the activity of ICAM-1-specific CAR T cells for patients with ICAM-1-positive TNBC [54].

4.4. Vaccines. The big advances of immunotherapy seen in TNBC led to push research about genetic vaccine against TNBC in vitro. It has been demonstrated that TNBC presents a high level of expression of Runx 2 in TNBC comparing to other breast cancer subtypes.

A Runx2 lentivirus transfection system was successfully engineered, and Runx2 was transduced into dendritic cells while maintaining stable expression. The sustained and stable cytotoxic $\mathrm{T}$ cells induced in the transfected group had higher and more specific antitumor efficacy against TNBC, compared with the other cell lines. With these results, Runx2 is an attractive target in TNBC and development of Runx2DC vaccine may induce specific and efficient activity in TNBC in vitro [55].

Cancer-testis antigens (CTAs) are a heterogeneous group of tumor-associated antigens (TAAs) present features that may be very attractive to be targeted by immunotherapeutic agents [56]. TNBC expresses several specific CTAs such as the MAGE group, SP17, and NY-ESO-1.

NY-ESO-1 expression was identified as an independent good prognostic factor in TNBC, and its expression was associated with higher humoral immune response and higher TILs [57]. Thus, the identification of tumors expressing NY-ESO-1 can allow the selection of patients with a higher potential of response to vaccination therapy.

SP17 is a protein expressed in breast cancer more importantly than in TNBC. The SP17-specific, HLA class I-restricted, cytotoxic $\mathrm{T}$ lymphocytes were associated with higher activity against breast cancer cells. Therefore, SP17 may be interesting in the development of breast cancer vaccines.

\section{Conclusion}

Immunotherapy has shown high efficacy in TNBC especially after the results from the IMpassion130 trial that has changed the standard of care in at least a subset of patients with metastatic PD-L1-positive TNBC. New strategies by the combination of immune checkpoint blockade with conventional therapies including chemotherapy, radiotherapy, and targeted therapies are also promising, and further research is needed to identify new biomarkers to select better responders to these treatments.

\section{Conflicts of Interest}

The authors declare that they have no conflicts of interest.

\section{References}

[1] B. D. Lehmann, J. A. Bauer, X. Chen et al., "Identification of human triple negative breast cancer subtypes and preclinical models for selection of targeted therapies," The Journal of Clinical Investigation, vol. 121, no. 7, pp. 2750-2767, 2011.

[2] R. Dent, M. Trudeau, K. I. Pritchard et al., "Triple-negative breast cancer: clinical features and patterns of recurrence," 
Clinical Cancer Research, vol. 13, no. 15, pp. 4429-4434, 2007.

[3] B. D. Lehmann, B. Jovanović, X. I. Chen et al., "Refinement of triple-negative breast cancer molecular subtypes: implications for neoadjuvant chemotherapy selection," PLoS One, vol. 11, no. 6, article e0157368, 2016.

[4] M. D. Burstein, A. Tsimelzon, G. M. Poage et al., "Comprehensive genomic analysis identifies novel subtypes and targets of triple-negative breast cancer," Clinical Cancer Research, vol. 21, no. 7, pp. 1688-1698, 2015.

[5] The Cancer Genome Atlas Network, "Comprehensive molecular portraits of human breast tumours," Nature, vol. 490, no. 7418, pp. 61-70, 2012.

[6] K. Oualla, H. M. el-Zawahry, B. Arun et al., "Novel therapeutic strategies in the treatment of triple-negative breast cancer," Therapeutic Advances in Medical Oncology, vol. 9, no. 7, pp. 493-511, 2017.

[7] I. Gingras, Azim HA Jr, M. Ignatiadis, and C. Sotiriou, "Immunology and breast cancer: toward a new way of understanding breast cancer and developing novel therapeutic strategies," Clinical Advances in Hematology \& Oncology, vol. 13, no. 6, pp. 372-382, 2015.

[8] Y. Wu, W. Chen, Z. P. Xu, and W. Gu, "PD-L1 distribution and perspective for cancer immunotherapy-blockade, knockdown, or inhibition," Frontiers in Immunology, vol. 10, pp. 2022-2236, 2019.

[9] S. Loi, "Tumor-infiltrating lymphocytes, breast cancer subtypes and therapeutic efficacy," Oncoimmunology, vol. 2, no. 7, article e24720, 2014.

[10] E. M. Ibrahim, M. E. Al-Foheidi, M. M. Al-Mansour, and G. A. Kazkaz, "The prognostic value of tumor-infiltrating lymphocytes in triple-negative breast cancer: a meta-analysis," Breast Cancer Research and Treatment, vol. 148, no. 3, pp. 467-476, 2014.

[11] S. Liu, J. Lachapelle, S. Leung, D. Gao, W. D. Foulkes, and T. O. Nielsen, "CD8 lymphocyte infiltration is an independent favorable prognostic indicator in basal-like breast cancer," Breast Cancer Research, vol. 14, no. 2, p. R48, 2012.

[12] E. A. Mittendorf, A. V. Philips, F. Meric-Bernstam et al., "PDL1 expression in triple negative breast cancer," Cancer Immunology Research, vol. 2, no. 4, pp. 361-370, 2014.

[13] M. Miyashita, H. Sasano, K. Tamaki et al., "Prognostic significance of tumor-infiltrating CD8+ and FOXP3+ lymphocytes in residual tumors and alterations in these parameters after neoadjuvant chemotherapy in triple-negative breast cancer: a retrospective multicenter study," Breast Cancer Research, vol. 17, no. 1, p. 124, 2015.

[14] M. E. Keir, M. J. Butte, G. J. Freeman, and A. H. Sharpe, "PD-1 and its ligands in tolerance and immunity," Annual Review of Immunology, vol. 26, no. 1, pp. 677-704, 2008.

[15] R. Nanda, L. Q. M. Chow, E. C. Dees et al., "Pembrolizumab in patients with advanced triple-negative breast cancer: phase Ib KEYNOTE-012 study," Journal of Clinical Oncology, vol. 34, no. 21 , pp. $2460-2467,2016$.

[16] S. Adams, P. Schmid, H. S. Rugo et al., "Phase 2 study of pembrolizumab (pembro) monotherapy for previously treated metastatic triple-negative breast cancer (mTNBC): KEYNOTE-086 cohort A," Journal of Clinical Oncology, vol. 35, 15_Supplement, pp. 1008-1008, 2017.

[17] S. Adams, S. Loi, D. Toppmeyer et al., "Phase 2 study of pembrolizumab as first-line therapy for PD-L1-positive metastatic triple-negative breast cancer (mTNBC): preliminary data from KEYNOTE-086 cohort B," Journal of Clinical Oncology, vol. 35, 15_Supplement, pp. 1088-1088, 2017.

[18] S. M. Tolaney, K. Kalinsky, V. Kaklamani, C. Savulsky, M. Olivo, and G. Aktan, "Phase 1b/2 study to evaluate eribulin mesylate in combination with pembrolizumab in patients with metastatic triple-negative breast cancer," Cancer Research, vol. 78, 4 Supplement, 2018.

[19] J. Cortés, O. Lipatov, S. Im et al., "KEYNOTE-119: phase 3 study of pembrolizumab versus single-agent chemotherapyfor metastatic triple-negative breast cancer," Annals of Oncology, vol. 30, Supplement_5, pp. v851-v934, 2019.

[20] J. Cortes, D. W. Cescon, H. S. Rugo et al., "KEYNOTE-355: randomized, double-blind, phase III study of pembrolizumab + chemotherapy versus placebo + chemotherapy for previously untreated locally recurrent inoperable or metastatic triple-negative breast cancer," Journal of Clinical Oncology, vol. 38, 15_Supplement, p. 1000, 2020.

[21] R. Nanda, M. C. Liu, C. C. Yau et al., "Pembrolizumab plus standard neoadjuvant therapy for high-risk breast cancer (BC): results from I-SPY 2," Journal of Clinical Oncology, vol. 35, 15_Supplement, p. 506, 2017.

[22] P. Schmid, J. Cortes, L. Pusztai et al., "Pembrolizumab for early triple-negative breast cancer," New England Journal of Medicine, vol. 382, no. 9, pp. 810-821, 2020.

[23] K. Mrleen, L. Voorwerk, H. Horlings et al., "Adaptive phase II randomized trial of nivolumab after induction treatment in triple negative breast cancer (TONIC trial): Final response data stage I and first translational data," Journal of Clinical Oncology, vol. 36, Supplement_15, pp. 1012-1012, 2018.

[24] L. A. Emens, C. Cruz, J. P. Eder et al., "Long-term clinical outcomes and biomarker analyses of atezolizumab therapy for patients with metastatic triple-negative breast cancer," JAMA Oncology, vol. 5, no. 1, pp. 74-82, 2019.

[25] P. Schmid, S. Adams, H. S. Rugo et al., "Atezolizumab and nab-paclitaxel in advanced triple-negative breast cancer," The New England Journal of Medicine, vol. 379, no. 22, pp. 21082121, 2018.

[26] "Atezolizumab and paclitaxel versus placebo and paclitaxel in participants with previously untreated locally advanced or metastatic triple negative breast cancer (TNBC) (IMpassion131)," Clinical http://Trials.gov.Identifier:NCT03125902.

[27] "Atezolizumab and chemotherapy compared with placebo and chemotherapy in the neoadjuvant setting in participants with early stage triple negative breast cancer (IMpassion031)," Clinical http://Trials.gov.Identifier:NCT03197935.

[28] L. Gianni, C. Huang, D. Egle et al., Pathologic complete response to neoadjuvant treatment with or without atezolizumab in triple-negative, early high-risk and locally advanced breast cancer. Neo TRIPAPDL1 Michelangelo randomized study, 2019 San Antonio Breast Cancer Symposium, 2019, Abstract GS3-04.

[29] U. Keilholz, J. M. Mehnert, S. Bauer et al., "Avelumab in patients with previously treated metastatic melanoma: phase $1 \mathrm{~b}$ results from the JAVELIN Solid Tumor trial," Journal for Immunotherapy of Cancer, vol. 7, no. 1, 2019.

[30] "Adjuvant treatment for high-risk triple negative breast cancer patients with the anti-PD-l1 antibody avelumab (A-BRAVE)," ClinicalTrials.gov Identifier: NCT02926196.

[31] "Pilot study of durvalumab and Vigil in advanced women's cancers," ClinicalTrials.gov Identifier: NCT02725489. 
[32] "Study of safety and efficacy of durvalumab in combination with paclitaxel in metastatic triple negative breast cancer patients," ClinicalTrials.gov Identifier: NCT02628132.

[33] "Phase I/II study of the anti-programmed death ligand-1 antibody MEDI4736 in combination with olaparib and/or cediranib for advanced solid tumors and advanced or recurrent ovarian, triple negative breast, lung, prostate and colorectal cancers," ClinicalTrials.gov Identifier: NCT02484404.

[34] "Tremelimumab in patients with advanced solid tumors," ClinicalTrials.gov Identifier: NCT02527434.

[35] "Epacadostat (INCB024360) in combination with durvalumab (MEDI4736) in subjects with selected advanced solid tumors (ECHO-203)," ClinicalTrials.gov Identifier: NCT02318277.

[36] S. Loibl, M. Untch, N. Burchardi et al., "A randomised phase II study investigating durvalumab in addition to an anthracycline taxane-based neoadjuvant therapy in early triplenegative breast cancer: clinical results and biomarker analysis of GeparNuevo study," Annals of Oncology, vol. 30, no. 8, pp. 1279-1288, 2019.

[37] L. Pusztai, E. W. Hofstatter, G. G. Chung et al., "Durvalumab (MEDI4736) concurrent with nab-paclitaxel and dose dense doxorubicin cyclophosphamide (ddAC) as neoadjuvant therapy for triple negative breast cancer (TNBC)," Journal of Clinical Oncology, vol. 36, 15_Supplement, pp. 586-586, 2018.

[38] R. M. Chabanon, M. Pedrero, C. Lefebvre, A. Marabelle, J. C. Soria, and S. Postel-Vinay, "Mutational landscape and sensitivity to immune checkpoint blockers," Clinical Cancer Research, vol. 22, no. 17, pp. 4309-4321, 2016.

[39] E. E. Parkes, S. M. Walker, L. E. Taggart et al., "Activation of STING-dependent innate immune signaling by S-phasespecific DNA damage in breast cancer," Journal of the National Cancer Institute, vol. 109, no. 1, article djw199, 2017.

[40] R. Chanut and V. Pétrilli, "Détection de l'ADN cytosolique par la voie cGAS-STING," Médecine/Sciences, vol. 35, no. 6-7, pp. 527-534, 2019.

[41] S. Vinayak, S. M. Tolaney, L. Schwartzberg et al., "Open-label clinical trial of niraparib combined with pembrolizumab for treatment of advanced or metastatic triple-negative breast cancer," JAMA Oncology, vol. 5, no. 8, article 1132, 2019.

[42] "Phase II Multicenter study of durvalumab and olaparib in platinum treated advanced triple negative breast cancer (DORA) (DORA)," ClinicalTrials.gov Identifier: NCT03167619.

[43] "Avelumab plus talazoparib in locally advanced or metastatic solid tumors," ClinicalTrials.gov Identifier: NCT03330405.

[44] "A combination study of rucaparib and atezolizumab in participants with advanced gynecologic cancers and triple-negative breast cancer," ClinicalTrials.gov Identifier: NCT03101280.

[45] "Olaparib with or without atezolizumab in treating patients with locally advanced unresectable or metastatic non-HER2-positive breast cancer," ClinicalTrials.gov Identifier: NCT02849496.

[46] S. Goel, M. J. DeCristo, A. C. Watt et al., "CDK4/6 inhibition triggers anti-tumour immunity," Nature, vol. 548, no. 7668, pp. 471-475, 2017.

[47] J. Deng, E. S. Wang, R. W. Jenkins et al., "CDK4/6 inhibition augments antitumor immunity by enhancing T-cell activation," Cancer Discovery, vol. 8, no. 2, pp. 216-233, 2018.

[48] Z. L. Teo, S. Versaci, S. Dushyanthen et al., "Combined CDK4/6 and PI3K $\alpha$ inhibition is synergistic and immunogenic in triple-negative breast cancer," Cancer Research, vol. 77, no. 22, pp. 6340-6352, 2017.
[49] H. S. Rugo, P. Kabos, M. N. Dickler et al., "Abstract P 1-09-01: a phase $1 \mathrm{~b}$ study of abemaciclib plus pembrolizumab for patients with hormone receptor-positive $(\mathrm{HR}+)$, human epidermal growth factor receptor 2-negative (HER2-) metastatic breast cancer (MBC)," Cancer Research, vol. 78, no. 4, article P1-09-01, 2018.

[50] R. Zhou, M. Yazdanifar, L. D. Roy et al., "CAR T cells targeting the tumor MUC1 glycoprotein reduce triple-negative breast cancer growth," Frontiers in Immunology, vol. 10, 2019.

[51] X. Xu, L. Zhang, X. He et al., "TGF- $\beta$ plays a vital role in triplenegative breast cancer (TNBC) drug-resistance through regulating stemness, EMT and apoptosis," Biochemical and Biophysical Research Communications, vol. 502, no. 1, pp. 160$165,2018$.

[52] G. Tozbikian, E. Brogi, K. Kadota et al., "Mesothelin Expression in Triple Negative Breast Carcinomas Correlates Significantly with Basal-Like Phenotype, Distant Metastases and Decreased Survival," PLoS One, vol. 9, no. 12, article e114900, 2014.

[53] J. Stagg and B. Allard, "Immunotherapeutic approaches in triple-negative breast cancer: latest research and clinical prospects," Therapeutic Advances in Medical Oncology, vol. 5, no. 3, pp. 169-181, 2013.

[54] P. Guo, J. Huang, L. Wang et al., "ICAM-1 as a molecular target for triple negative breast cancer," Proceedings of the National Academy of Sciences of the United States of America, vol. 111, no. 41, pp. 14710-14715, 2014.

[55] M. Tang, Y. Liu, Q.-. C. Zhang et al., "Antitumor efficacy of the Runx2-dendritic cell vaccine in triple-negative breast cancer in vitro," Oncology Letters, vol. 16, no. 3, pp. 2813-2822, 2018.

[56] A. M. Mahmoud, "Cancer testis antigens as immunogenic and oncogenic targets in breast cancer," Immunotherapy, vol. 10, no. 9, pp. 769-778, 2018.

[57] H. J. Lee, J. Y. Kim, I. H. Song, I. A. Park, J. H. Yu, and G. Gong, "Expression of NY-ESO-1 in triple-negative breast cancer is associated with tumor-infiltrating lymphocytes and a good prognosis," Oncology, vol. 89, no. 6, pp. 337-344, 2015. 